F. Gomez et D. Lehmann

Nagoya Math. J.

Vol. 135 (1994), 197-206

\title{
DES DERIVEES DE RESIDUS ET UNE IDENTITE REMARQUABLE
}

\section{FRANCISCO GOMEZ ${ }^{*}{ }_{\text {ET }}$ DANIEL LEHMANN **)}

\section{Introduction}

Soit $\varphi$ un polynome symétrique de $q$ variables $(q \geq 1)$, homogène de degré $p(p$ entier $\geq 2)$, à coefficients complexes. Soient $\left(\alpha_{1}, \alpha_{2}, \ldots, \alpha_{p}\right)$ et $\left(\beta_{1}, \beta_{2}, \ldots, \beta_{p}\right)$ deux familles de $p$ nombres complexes, tous non nuls, et telles que toutes les différences $\frac{\beta_{j}}{\beta_{i}}-\frac{\alpha_{j}}{\alpha_{i}}$ soient également non nulles pour $i \neq j$. Soient enfin $\left(\lambda_{1}, \lambda_{2}, \ldots, \lambda_{q}\right)$ et $\left(\mu_{1}, \mu_{2}, \ldots, \mu_{q}\right)$ deux familles de $q$ nombres complexes. On a alors:

THÉORÈME 1.

$$
\frac{\varphi\left(\mu_{1}, \ldots, \mu_{q}\right)}{\Pi_{i=1}^{p} \beta_{i}}-\frac{\varphi\left(\lambda_{1}, \ldots, \lambda_{q}\right)}{\Pi_{i=1}^{p} \alpha_{i}}=\sum_{i=1}^{p} \frac{\varphi\left(\frac{\mu_{1}}{\beta_{i}}-\frac{\lambda_{1}}{\alpha_{i}}, \ldots, \frac{\mu_{q}}{\beta_{i}}-\frac{\lambda_{q}}{\alpha_{i}}\right)}{\Pi_{j, j \neq i}\left(\frac{\beta_{j}}{\beta_{i}}-\frac{\alpha_{j}}{\alpha_{i}}\right)} .
$$

Si les $\alpha_{i}(1 \leq i \leq p)$ et les $\lambda_{u}(1 \leq u \leq q)$ dépendent maintenant différentiablement (resp. holomorphiquement) d'un paramètre réel (resp. complexe) $t$, on en déduit immédiatement, en notant' la dérivée par rapport à $t$, et en supposant non nuls tous les nombres figurant en dénominateur, le

COROLLAiRE.

$$
\left(\frac{\varphi\left(\lambda_{1}, \ldots, \lambda_{q}\right)}{\Pi_{i=1}^{p} \alpha_{i}}\right)^{\prime}=\sum_{i=1}^{p} \frac{\varphi\left(\left(\frac{\lambda_{1}}{\alpha_{i}}\right)^{\prime}, \ldots,\left(\frac{\lambda_{q}}{\alpha_{i}}\right)^{\prime}\right)}{\Pi_{j, j \neq i}\left(\frac{\alpha_{j}}{\alpha_{i}}\right)^{\prime}}
$$

Received September 22, 1993.

* Partially supported by a DGICYT grant (PB91-0412) and a Junta de Andalucía grant (1197)

**) CNRS (UA 1407) 
Dans cet article, nous allons d'abord démontrer le théorème 1 comme un cas particulier, correspondant à des cas de "non dégénérescence", en un sens voisin de celui de Bott ([B1]), d'une formule de différence de deux résidus de Grothendieck (théorème 2 ci-dessous). Le cas $\left(p=q, \alpha_{i}=\lambda_{i}, \beta_{i}=\mu_{i}\right)$ a déjà été démontré implicitement dans [L2]. Dans la généralisation qui suit, nous nous efforcerons d'éviter toute considération globale et de rester au voisinage d'un point où tous les fibrés sont triviaux: malgré celà, nous n'avons pas réussi à nous passer de ces outils usuels de la Géométrie différentielle que sont les connexions et la théorie de Chern-Weil (nous faisons en particulier un usage intensif des opérateurs de différence itérées de Bott pour une famille de connexions, dont la traduction naïve en termes de coordonnées locales nous semble beaucoup trop compliquée dans le cas général pour qu'elle vaille la peine d'être faite).

Nous donnerons ensuite, de l'identité remarquable du théorème 1, une démonstration purement algébrique et beaucoup plus élémentaire. [Notons toutefois que c'est par la première méthode que cette identité a été découverte!]

Merci à M. Soares pour de stimulantes conversations. C'est en particulier lui qui a suggéré d'utiliser le théorème 1 pour obtenir une formule de dérivation. Il l'a d'ailleurs utilisée dans [S] pour étudier les systèmes différentiels algébriques sur $\mathbf{P}^{\mathrm{n}}(\mathbf{C})$ qui n'admettent pas de sous variété algébrique invariante.

\section{Enoncé du théorème 2}

Comme dans l'introduction, notons $\varphi$ un polynome symétrique de $q$ variables $(q \geq 1)$, homogène de degré $p$ ( $p$ entier $\geq 2)$, à coefficients complexes (qu'on peut aussi étendre, d'après ([B2]), en un polynome homogène de degré $p$ par rapport aux coefficients des matrices $q \times q$ coefficients complexes, invariant par la représentation adjointe de $G L(q, \mathbf{C}),\left(\lambda_{1}, \ldots, \lambda_{q}\right)$ étant identifié à la matrice diagonale correspondante). Soient $U$ un ouvert de $\mathbf{C}^{p}$ (coordonnées complexes naturelles notées $\left.\left(z_{i}\right)_{i}\right), X=\sum_{i=1}^{p} X_{i} \frac{\partial}{\partial z_{i}}$ et $Y=\sum_{i=1}^{p} Y_{i} \frac{\partial}{\partial z_{i}}$ deux champs de vecteurs holomorphes sur $U$ s'annulant tous deux en le même point $m_{0}$ de $U$, seulement en ce point, et commutant, (c'est dire définissant une action holomorphe infinitésimale du groupe $\mathbf{C}^{2}$ sur $U:[X, Y]=0$ ). Soient $M$ et $N$ deux applications holomorphes de $U$ dans l'espace des matrices $q \times q$ coefficients complexes. Nous allons évaluer la différence 


$$
\left[\begin{array}{c}
\varphi(N) d x_{1} \wedge d x_{2} \wedge \cdots d x_{p} \\
Y_{1}, Y_{2}, \ldots, Y_{p}
\end{array}\right]_{m_{0}}-\left[\begin{array}{c}
\varphi(M) d x_{1} \wedge d x_{2} \wedge \cdots d x_{p} \\
X_{1}, X_{2}, \ldots, X_{p}
\end{array}\right]_{m_{0}}
$$

en fonction du seul comportement de $(X, M)$ et $(Y, N)$ au voisinage des points de $U-\left\{m_{0}\right\}$ où $X$ et $Y$ sont colinéaires. [On a noté:

$$
\left[X_{1}, X_{2}, \ldots, X_{p}\right]_{m_{0}}=\int_{R} \frac{\eta}{\prod_{i=1}^{p} X_{i}}
$$

la valeur en $\left\{m_{0}\right\}$ du symbole résidu de Grothendieck, où $\eta$ désigne une $p$-forme holomorphe sur $U, X=\left(X_{1}, X_{2}, \ldots, X_{p}\right): U \rightarrow \mathbf{C}^{p}$ une application holomorphe, n'admettant qu'un seul zéro $\left\{m_{0}\right\}$ dans $U$, et $R$ désigne une certaine variété de dimension réelle $p$ incluse dans l'ensemble des points de $U$ où aucune des coordonnées $X_{i}$ ne s'annule et qu'on peut prendre égale (cf. [L1] section 6) à l'intersection de $p$ hypersurfaces d'equation $\left|X_{\imath}\right|=\varepsilon_{\imath}$, ( $\varepsilon_{i}$ désignant une constante positive) lorsque celles ci sont en position générale.

Plus précisément, notons $\Gamma$ l'ensemble (analytique) des points de $U-\left\{m_{0}\right\}$ où $X$ et $Y$ sont colinéaires, $S^{2 p-1}$ une $2 p-1$ sphère dans $U$ entourant $\left\{m_{0}\right\},\left(W_{h}\right)_{h}$ la famille des composantes connexes de $\Gamma \cap S^{2 p-1}$, et $\mathscr{T}_{h}$ une sous variété à bord de dimension $2 p-1$ dans $S^{2 p-1}$, contenant $W_{h}$ en son intérieur. Puisque $X$ et $Y$ commutent, $\Gamma$ est nécessairement invariant par l'action infinitésimale de $\mathbf{C}^{2}$ qu'ils engendrent: $X$ et $Y$ sont donc tangents ${ }^{(1)}$ à $\Gamma$.

On supposera (c'est la situation générique!) que $\Gamma$ est une courbe complexe singulière sans singularité en dehors de $\left\{m_{0}\right\}$, et que les différentes branches $\mathscr{B}_{h}$ de $\Gamma$ passant par $\left\{m_{0}\right\}$ sont transverses à $S^{2 p-1}$, (de sorte que chaque intersection $W_{h}=\mathscr{B}_{h} \cap S^{2 p-1}$ est un cercle).

Nous ferons enfin l':

Hypothèse $\left({ }^{*}\right)$ : Pour tout $h$, il existe un système de coordonées complexes $\left(x_{1}, y_{2}, \ldots, y_{p}\right)$ au voisinage de $\mathscr{T}_{h}$, tel que $W_{h}$ soit défini par les équations $y_{u}=0,(u=2, \ldots, p)$, tel que la restriction à $\mathscr{T}_{h}$ de la projection $\pi:\left(x, y_{2}, \ldots, y_{p}\right) \rightarrow x$ soit une fibration localement triviale, et tel que l'on ait:

(1) C'est en fait la seule hypothèse dont nous ayions réellement besoin, et il n'est pas nécessaire que $X$ et $Y$ commutent pour celà: par exemple, elle reste vérifiée si $X$ et $Y$ proviennent de l'action infinitésimale de l'algèbre de Lie complexe non commutative de dimension 2 (algèbre de Lie du groupe affine de $\mathbf{C}$ ). 
$A C \neq 0 \operatorname{sur}_{h}$, et $B_{u}=D_{u}=0$ sur $W_{h}(u=2, \ldots, p)$,

où l'on a posé: $X=A \frac{\partial}{\partial x}+\sum_{u=2}^{p} B_{u} \frac{\partial}{\partial y_{u}}$, et $Y=C \frac{\partial}{\partial x}+\sum_{u=2}^{p} D_{u} \frac{\partial}{\partial y_{u}}$.

THÉORÈmE 2. Sous l'hypothèse $\left({ }^{*}\right)$ on a la formule:

$$
\begin{gathered}
{\left[\begin{array}{c}
\varphi(N) d x_{1} \wedge d x_{2} \wedge \cdots d x_{p} \\
Y_{1}, Y_{2}, \ldots, Y_{p}
\end{array}\right]_{m_{0}}-\left[\begin{array}{c}
\varphi(M) d x_{1} \wedge d x_{2} \wedge \cdots d x_{p} \\
X_{1}, X_{2}, \ldots, X_{p}
\end{array}\right]_{m_{0}}=} \\
\frac{(-1)^{p-1}}{2 i \pi} \sum_{h} \int_{W_{h}}\left[\begin{array}{c}
\varphi\left(M_{h}\right) d y_{2} \wedge \cdots d y_{p} \\
Z_{2}, \ldots, Z_{p}
\end{array}\right]_{x} d x .
\end{gathered}
$$

où $\quad Z_{u}=\frac{D_{u}}{C}-\frac{B_{u}}{A}(2 \leq u \leq p), M_{h}=\frac{1}{C} N-\frac{1}{A} M$ et $\left[\begin{array}{c}\varphi\left(M_{h}\right) d y_{2} \wedge \cdots d y_{p} \\ Z_{2}, \ldots, Z_{p}\end{array}\right]_{x}$ représente la valeur en $x \in W_{h}$ du symbole résidu de Grothendieck sur la fibre $\pi^{-1}(x)$.

Remarque. On peut déduire du théorème 2 une formule de dérivation généralisant celle du corollaire du théorème 1 de la façon suivante. On suppose que les composantes $X_{i}$ du champ holomorphe $X$ ainsi que la matrice $M$ dépendent différentiablement (resp. holomorphiquement) d'un paramètre réel (resp. complexe) $t$. On note maintenant $\Gamma$ le lieu des points où $X$ et $\frac{\partial X}{\partial t}$ sont linéairement dépendants, et l'on fait des hypothèses analogues à celles du théorème 2 en remplaçant partout $X$ et $Y$ par $X$ et $\frac{\partial X}{\partial t}$. Les composantes $A$ et $B_{u}$ de $X$ sur $\mathscr{T}_{h}$ par rapport aux coordonnées $\left(x_{1}, y_{2}, \ldots, y_{p}\right)$ dépendent différentiablement (resp. holomorphiquement) de $t$. On a alors la formule:

$$
\begin{gathered}
\frac{\partial}{\partial t}\left[\begin{array}{c}
\varphi(M) d x_{1} \wedge d x_{2} \wedge \cdots d x_{p} \\
X_{1}, X_{2}, \ldots, X_{p}
\end{array}\right]_{m_{0}}= \\
\frac{(-1)^{p-1}}{2 i \pi} \sum_{h} \int_{W_{h}}\left[\begin{array}{c}
\varphi\left(\frac{\partial}{\partial t}\left(\frac{M}{A}\right)\right) d y_{2} \wedge \cdots d y_{p} \\
\frac{\partial}{\partial t}\left(\frac{B_{2}}{A}\right), \ldots, \frac{\partial}{\partial t}\left(\frac{B_{p}}{A}\right)
\end{array}\right]_{x} d x .
\end{gathered}
$$

Cas particulier. Supposons, avec les notations et hypothèses du théorème 1 , que l'on prenne: $X=\sum_{i=1}^{p} \alpha_{i} z_{i} \frac{\partial}{\partial z_{i}}, Y=\sum_{i=1}^{p} \beta_{i} z_{i} \frac{\partial}{\partial z_{i}}$ sur $\mathbf{C}^{\mathrm{p}}, m_{0}=0, \quad M=$ 
matrice diagonale $\left(\lambda_{1}, \ldots, \lambda_{q}\right)$, et $N=$ matrice diagonale $\left(\mu_{1}, \ldots, \mu_{q}\right)$. Les différentes branches de $\Gamma$ sont alors les droites complexes $\mathscr{B}_{i}(1 \leq i \leq p)$ définies par $\left(z_{j}=0\right.$ pour tout $\left.j \neq i\right)$, et l'on peut prendre comme coordonnées vérifiant l'hypothèse $\left({ }^{*}\right)$ au voisinage du cercle $W_{i}$ les mêmes coordonnées naturelles de $\mathbf{C}^{\mathrm{p}}$ réordonnées ainsi $x=z_{i}$ et $\left(y_{u}\right)_{u}=\left(z_{j}\right)_{j, j \neq i}$. Le théorème 2 fournit alors l'identité $\mathrm{du}$ théorème 1 .

\section{Démonstration du théorème 2}

Elle va se faire en plusieurs étapes:

1) Exhiber une $2 p-1$ forme $\xi$ sur $S^{2 p-1}$ telle que

$$
\left[\begin{array}{c}
\varphi(N) d x_{1} \wedge d x_{2} \wedge \cdots d x_{p} \\
Y_{1}, Y_{2}, \ldots, Y_{p}
\end{array}\right]_{m_{0}}-\left[\begin{array}{c}
\varphi(M) d x_{1} \wedge d x_{2} \wedge \cdots d x_{p} \\
X_{1}, X_{2}, \ldots, X_{p}
\end{array}\right]_{m_{0}}=\int_{S^{2 p-1}} \xi
$$

2) Exhiber une $2 p-2$ forme $\phi_{h}$ sur chaque $\partial \mathscr{T}_{h}$ telle que

$$
\int_{S^{2 p-1}} \xi=\sum_{h} \int_{\partial \mathcal{T}_{h}} \phi_{h}
$$

3) Montrer que

$$
\int_{\partial \mathscr{T}_{h}} \phi_{h}=\frac{(-1)^{p-1}}{2 i \pi} \int_{W_{h}}\left[\begin{array}{c}
\varphi\left(M_{h}\right) d y_{2} \wedge \cdots d y_{p} \\
Z_{2}, \ldots, Z_{p}
\end{array}\right]_{x} d x
$$

Soit $\left(\sigma_{1}, \ldots, \sigma_{q}\right)$ la base naturelle du module des sections holomorphes du fibré trivial $\mathbf{C}^{q} \times U \rightarrow U$. D'une connexion $\omega$ sur ce fibré trivial, nous dirons qu'elle est "adaptée à $(X, M)$ " si elle est définie par une loi de dérivation covariante vérifiant, relativement à la trivialisation précédente:

$$
\left\{\begin{array}{l}
\nabla_{X} \sigma_{u}=\left\langle M, \sigma_{u}\right\rangle, \\
\nabla_{Z} \sigma_{u}=0 \quad \forall Z \in T^{01} \text { (c'est à dire pour tout } Z \text { antiholomorphe). }
\end{array}\right.
$$

Notons $\Delta_{\omega_{0} \omega_{1} \cdots \omega_{r}}(\varphi)$ les opérateurs de différence itérée de Bott ([B3]), qui vérifient:

$(* *)$

$$
d \cdot \Delta_{\omega_{0} \omega_{1} \cdots \omega_{r}}(\varphi)=\sum_{j=0}^{r}(-1)^{j} \Delta_{\omega_{0} \cdots \hat{\omega}_{j} \cdots \omega_{r}}(\varphi)
$$

Si les connexions $\omega_{0}, \ldots, \omega_{r}$ sont toutes adaptées à $(X, M)$, on a alors:

$(* * *)$

$$
\Delta_{\omega_{0} \cdots \omega_{r}}(\varphi)=0 \text {. }
$$


$1^{\text {ère }}$ étape.

Si $\omega_{0}$ désigne la connexion canonique triviale, il a été démontré (lemme 5-3 du théorème 1' de [L1]):

$$
\left[\begin{array}{c}
\varphi(M) d x_{1} \wedge d x_{2} \wedge \cdots d x_{p} \\
X_{1}, X_{2}, \ldots, X_{p}
\end{array}\right]_{m_{0}}=\int_{S^{2 p-1}} \Delta_{\omega_{0} \omega}(\varphi) .
$$

Définissant de même $\omega^{\prime}$ relativement à $(Y, N)$, on peut donc prendre pour $\xi$ la forme $\Delta_{\omega \omega^{\prime}}(\varphi)$, puisque $\Delta_{\omega \omega^{\prime}}(\varphi)$ et $\Delta_{\omega_{0} \omega^{\prime}}(\varphi)-\Delta_{\omega_{0} \omega}(\varphi)$ sont cohomologues d'après $\left({ }^{*}\right)$.

\section{$2^{\grave{e ̀ m e}}$ étape.}

Il est possible de choisir les connexions $\omega$ et $\omega^{\prime}$ de telle sorte qu'au voisinage de chaque $\mathscr{T}_{h}$, elles soient aussi adaptées à $\left(\frac{\partial}{\partial y_{u}}\right.$, matrice 0$)$ pour tout $2 \leq u \leq p$, d'où: $\Delta_{\omega \omega^{\prime}}(\varphi)=0$ sur chaque $\mathscr{T}_{h}$. Puisque, $X$ et $Y$ sont linéairement indépendants en dehors de $\Gamma$, il existe une connexion $\omega_{1}$ au voisinage de $S^{2 p-1}-\cup_{h} \mathscr{T}_{h}$, adaptée à la fois à $(X, M)$ et $(Y, N)$, de sorte que l'on a alors:

$\Delta_{\omega_{1} \omega}(\varphi)=\Delta_{\omega_{1} \omega^{\prime}}(\varphi)=0$ d'après $(* * *)$, et $\Delta_{\omega \omega^{\prime}}(\varphi)=d \Delta_{\omega_{1} \omega \omega^{\prime}}(\varphi)$ d'après $\left({ }^{*}\right)$. On en déduit, appliquant la formule de Stokes, que l'on peut prendre pour $\phi_{h}$ la restriction de $-\Delta_{\omega_{1} \omega \omega^{\prime}}(\varphi)$ à $\partial \mathscr{T}_{h}$.

$3^{\text {ème }}$ étape.

Puisque $X$ and $Y$ sont linéairement indépendants sur $\partial \mathscr{T}_{h}$, l'une au moins des fonctions $Z_{u}$ ne s'annule pas sur cette variété: par conséquent les ouverts $O_{u}$ de $\partial \mathcal{T}_{h}$ définis par $Z_{u} \neq 0$ constituent un recouvrement ouvert $\mathscr{O}$ de $\partial \mathcal{T}_{h}$. Soit $\left(R_{2}^{h}, \ldots\right.$, $R_{p}^{h}$ ) un système d'alvéoles subordonné à ce recouvrement, au sens où nous l'avons défini dans ([L1] section 1). Posons: $R_{2, \cdots, p}^{h}=\cap_{\lambda=2}^{p} R_{\lambda}^{h}$. Nous ferons aussi l'hypothèse, facile à rèaliser en pratique, que $R_{2, \cdots, p}^{h}$ fibre au dessus de $W_{h}$. Pour démontrer que $\int_{\partial \mathscr{T}_{h}} \phi_{h}=\frac{(-1)^{p-1}}{2 i \pi} \int_{W_{h}}\left[\begin{array}{c}\varphi\left(M_{h}\right) d y_{2} \wedge \cdots d y_{p} \\ Z_{2}, \ldots, Z_{p}\end{array}\right]_{x} d x$, il nous suffit, compte tenu du théorème 5 de [L1] (section 6), et du "théorème de Fubini" $\left(\int_{R_{2, \ldots, p}^{h}}=\int_{W_{h}} \circ f\right.$, où $f$ désigne l'integration le long de la fibre $)$, de prouver le

LEMME 1. Les formes $\phi_{h}=-\Delta_{\omega_{1} \omega \omega^{\prime}}(\varphi)$ sur $\partial \mathscr{T}_{h}$, et $\frac{\varphi\left(M_{h}\right) d x \wedge d y_{2} \wedge \ldots d y_{p}}{\Pi_{u=2}^{p} Z_{u}}$ sur l'intersection des ouverts $O_{u}$ dans $\partial \mathcal{T}_{h}$, sont cohomologues, lorsqu'elles sont toutes 
deux considérées comme appartenant au complexe total de $\tilde{C} e c h$-de Rham $C D R^{*}(\mathscr{O})$ associé au recouvrement $\mathfrak{O}$.

Démonstration. Au voisinage de chaque $O_{u}$, les $p$ champs de vecteurs $X, Y, \frac{\partial}{\partial y_{2}}, \ldots, \frac{\partial}{\partial y_{p}}$ sont linéairement indépendants; il $\mathrm{y}$ exists donc une connexion $\eta_{u}$ (d'ailleurs unique) adaptée simultanément à $(X, M),(Y, N)$, et à tous les $\left(\frac{\partial}{\partial y_{v}}, 0\right)(v \neq u)$.

Pour tout $k$ simplexe $L=\left(l_{0} \cdots l_{k}\right)$ dans le nerf de $\mathscr{O}$, et toute famille de connexions $\left(\omega_{j_{1}}, \ldots, \omega_{j_{s}}\right)$, posons: $\Delta_{\omega_{j_{1}} \cdots \omega_{j_{s}} \eta_{L}}=\Delta_{\omega_{j_{1}} \cdots \omega_{j_{s}} \eta_{l_{0}} \cdots \eta_{l_{k}}}(\varphi)$, et définissons $\gamma \in C D R^{2 p-3}(\mathscr{O})$ comme la famille $\left(\gamma_{L}\right)_{L}$ donnée par:

$\gamma_{L}=(-1)^{\left[\frac{k+1}{2}\right]} \Delta_{\omega_{1} \omega \omega^{\prime} \eta_{L}}$, où $k$ désigne la dimension $\|L\|$ du simplexe $L$.

La différentielle totale $D \gamma$ de $\gamma$ dans $C D R^{*}(\mathscr{O})$ est alors donnée par:

$$
\begin{aligned}
D \gamma_{L}= & (-1)^{\left[\frac{k+1}{2}\right]+k}\left(\Delta_{\omega \omega^{\prime} \eta_{L}}-\Delta_{\omega_{1} \omega^{\prime} \eta_{L}}+\Delta_{\omega_{1} \omega \eta_{L}}+\sum_{\alpha=0}^{\mathrm{k}}(-1)^{\alpha+1} \Delta_{\omega_{1} \omega \omega^{\prime}, \eta_{L-l_{\alpha}}}\right) \\
& +\sum_{\alpha=0}^{k}(-1)^{\left[\frac{k}{2}\right]+\alpha} \Delta_{\omega_{1} \omega \omega^{\prime} \eta_{L-l_{\alpha}}} \\
= & (-1)^{\left[\frac{k+1}{2}\right]+k}\left(\Delta_{\omega \omega^{\prime} \eta_{L}}-\Delta_{\omega_{1} \omega^{\prime} \eta_{L}}+\Delta_{\omega_{1} \omega \eta_{L}}\right) \text { pour }\|L\| \geq 1,
\end{aligned}
$$

and $D \gamma_{l}=\Delta_{\omega \omega^{\prime} \eta_{l}}-\Delta_{\omega_{1} \omega \eta_{l}}-\Delta_{\omega_{1} \omega \omega^{\prime}}$ pour $\|L\|=0$.

Mais, d'après $\left({ }^{* * *}\right), \Delta_{\omega \omega^{\prime} \eta_{L}}=0$ sauf pour $L=(2,3, \ldots, p)$, c'est à dire sauf pour $|L|=p-2$. En effet, pour $|L|=p-2$, il existe au moins 1 indice $u$ tel que chacune des connexions $\omega, \omega^{\prime}, \eta_{l}(l \in L)$ soit adaptée à $\left(\frac{\partial}{\partial y_{u}}, 0\right)$.

De même, $\Delta_{\omega_{1} \omega \eta_{L}}=0$ (resp. $\Delta_{\omega_{1} \omega^{\prime} \eta_{L}}=0$, parce que $\omega_{1}$, $\omega$ et $\eta_{l}(l \in L)$ (resp. $\omega_{1}, \omega^{\prime}$ et $\left.\eta_{l}\right)$ sont toutes adaptées à $(X, M)$ (resp. $(Y, N)$ ). Ainsi, dans l'expression de $D \gamma$, tous les termes sont nuls, sauf $-\Delta_{\omega_{1} \omega \omega^{\prime}}$ dans chaque terme $(D \gamma)_{l}$, et $(-1)^{\left[\frac{p}{2}\right]+1} \Delta_{\omega \omega^{\prime} \eta_{(2,3, \cdots, p)}}$ dans $(D \gamma)_{(2,3, \cdots, p)}$.

Les formes $-\Delta_{\omega_{1} \omega \omega^{\prime}}(\varphi)$ et $(-1)^{\left[\frac{p}{2}\right]+1} \Delta_{\omega \omega^{\prime} \eta_{(2,3, \cdots, p)}}$ sont donc cohomologues dans $C D R^{*}(\mathscr{O})$. Il ne reste plus quà montrer le:

LEMME 2 .

$$
(-1)^{\left[\frac{p}{2}\right]} \Delta_{\omega \omega^{\prime} \eta_{(2,3, \cdots, p)}}=\frac{\varphi\left(M_{h}\right) d x \wedge d y_{2} \wedge \cdots d y_{p}}{\Pi_{u=2}^{p} Z_{u}}
$$


Relativement à la trivialisation naturelle $\left(\sigma_{1}, \ldots, \sigma_{q}\right)$, on a : $\omega=\frac{1}{A} M d x$ et $\omega^{\prime}=\frac{1}{C} N d x$, d'où $\omega^{\prime}-\omega=M_{h} d x$.

D'autre part la condition imposée à $\eta_{u}$, d'être simultanément adaptée à $(X, M)$, $(Y, N)$, et à tous les $\left(\frac{\partial}{\partial y_{v}}, 0\right)(v \neq u)$ s'écrit:

$$
\eta_{u}=\frac{1}{Z_{u}}\left(\frac{1}{A C}\left(D_{u} M-B_{u} N\right) d x+M_{h} d y_{u}\right)
$$

La combinaison affine $\tilde{\omega}$ de ces $p+1$ connexions au voisinage de $\left(\cap_{u=2}^{p} \mathscr{O}_{u}\right) \times \Delta^{p}$ est alors égale à

$$
\tilde{\omega}=\left(t \frac{1}{A} M+t^{\prime} \frac{1}{C} N+\sum t^{\prime \prime}{ }_{u} Q_{u}\right) \wedge d x+\left(\sum_{\lambda} \frac{t^{\prime \prime}{ }_{u} d y_{u}}{Z_{u}}\right) M_{h}
$$

pour une certaine matrice $Q_{u}$, avec $\left(t+t^{\prime} \sum_{u} t^{\prime \prime}{ }_{u}\right) \equiv 1$, et $t, t^{\prime}, t^{\prime \prime}{ }_{u}$ tous $\geq 0$. Sa courbure peut alors s'écrire:

$$
\tilde{\Omega}=\left(d t \frac{1}{A} M+d t^{\prime} \frac{1}{C} N+\sum d t^{\prime \prime}{ }_{u} Q_{u}\right) \wedge d x+\left(\sum_{u} \frac{t^{\prime \prime}{ }_{u} \wedge d y_{u}}{Z_{u}}\right) M_{h}+Q \wedge d x,
$$

où $Q$ ne contient aucun terme en $d t, d t^{\prime}$ ou $d t^{\prime \prime}{ }_{u}$. Puisque $d t^{\prime}=-d t$ (modulo $\left.d t^{\prime \prime}{ }_{u}\right)$, et puisqu'il n'y a que $p-1 d t^{\prime \prime}{ }_{u}$ distincts, $\Delta_{\tilde{\omega}}(\varphi)=\varphi(\tilde{\Omega}, \tilde{\Omega}, \ldots, \tilde{\Omega})$ peut s'écrire:

$$
\frac{(-1)^{\left[\frac{p}{2}\right]}(p !)}{\Pi_{u} Z_{u}} \varphi\left(M_{h}\right)\left(d t \wedge d t^{\prime \prime}{ }_{2} \wedge \cdots \wedge d t^{\prime \prime}{ }_{p}\right) \wedge d x \wedge\left(d y_{2} \wedge \cdots \wedge d y_{p}\right)
$$

+ une expression avec au plus $p-1$ termes en $d t, d t^{\prime \prime}{ }_{u}$.

Par intégration sur $\Delta^{p}$, et utilisant l'égalité

$$
\int_{\Delta^{p}} d t_{1} \wedge d t_{2}^{\prime} \wedge \cdots \wedge d t_{p}^{\prime}=\frac{1}{p !}
$$

on obtient la formule du lemme 2, QED.

\section{Deuxième démonstration du théorème 1 , purement algébrique}

Lemme 3. Soit $\left(\rho_{1}, \rho_{2}, \ldots, \rho_{p}\right)$ une famille arbitraire de $p$ nombres complexes tous distincts et non nuls. On a alors, pour $p \geq 1$ et pour tout entier $k$ compris entre 0 et $p$ : 


$$
\sum_{i=1}^{p} \frac{\rho_{i}^{k-1}}{\Pi_{j, j \neq i}\left(\rho_{j}-\rho_{i}\right)}=\left\{\begin{array}{l}
0 \text { pour } 1 \leq k \leq p-1 \\
(-1)^{p+1} \text { pour } k=p \\
\frac{1}{\Pi_{i=1}^{p} \rho_{i}} \text { pour } k=0
\end{array}\right.
$$

Démonstration. Le déterminant

$$
D_{k}=\operatorname{det}\left(\begin{array}{cccc}
1 & 1 & \cdots & 1 \\
\rho_{1} & \rho_{2} & \cdots & \rho_{p} \\
\cdots & \cdots & \cdots & \cdots \\
\rho_{1}^{p-2} & \rho_{2}^{p-2} & \cdots & \rho_{p}^{p-2} \\
\rho_{1}^{k-1} & \rho_{2}^{k-1} & \cdots & \rho_{p}^{k-1}
\end{array}\right)
$$

est évidemment égal à

$$
\left\{\begin{array}{l}
0 \text { pour } 1 \leq k \leq p-1,(\text { car } 2 \text { lignes sont égales }) \\
\prod_{j>i}\left(\rho_{j}-\rho_{i}\right) \text { pour } k=p \text { (déterminant de Van der Monde) } \\
\frac{(-1)^{p+1}}{\Pi_{i=1}^{p} \rho_{i}} D_{p}=\frac{(-1)^{p+1}}{\Pi_{i=1}^{p} \rho_{i}} \prod_{j>i}\left(\rho_{j}-\rho_{i}\right) \text { pour } k=0
\end{array}\right.
$$

D'autre part, un dévelopement par rapport à la dernière ligne donne:

$$
D_{k}=\sum_{i=1}^{p}(-1)^{p+i}\left(\rho_{i}^{k-1} \prod_{r>s, r \neq i, s \neq i}\left(\rho_{r}-\rho_{s}\right)\right),
$$

d'où le lemme 3 en égalant les deux expressions.

Lemme 4. Soit $\varphi$ un polynome symétrique de $q$ variables $(q \geq 1)$, homogène de degré $p$ ( $p$ entier $\geq 2$ ), à coefficients complexes. Soient $\rho_{1}, \rho_{2}, \ldots, \rho_{p}$ comme au lemme 3. Soient enfin $\left(\lambda_{1}, \lambda_{2}, \ldots, \lambda_{q}\right)$ et $\left(\mu_{1}, \mu_{2}, \ldots, \mu_{q}\right)$ deux familles de $q$ nombres complexes. On a alors:

$$
\frac{\varphi\left(\mu_{1}, \ldots, \mu_{q}\right)}{\Pi_{i=1}^{p} \rho_{i}}-\varphi\left(\lambda_{1}, \ldots, \lambda_{q}\right)=\sum_{i=1}^{p} \frac{\varphi\left(\mu_{1}-\lambda_{1} \rho_{i}, \ldots, \mu_{q}-\lambda_{q} \rho_{i}\right)}{\rho_{i} \Pi_{j, j \neq i}\left(\rho_{j}-\rho_{i}\right)} .
$$

Démonstration. Soit $\Phi$ le polynome sur l'espace des matrices $q \times q$ à coefficients complexes, invariant par la représentation adjointe de $G L(q, \mathbf{C})$, qui, à la matrice $K$ de valeurs propres $\left(\lambda_{1}, \ldots, \lambda_{q}\right)$, associe le nombre $\Phi(K)=\varphi\left(\lambda_{1}, \ldots, \lambda_{q}\right)$, et soit $\tilde{\Phi}$ la forme $p$-multilinéaire symétrique associée à $\Phi$ par polarisation: $\Phi(K)=\frac{1}{p !} \tilde{\Phi}(K, K, \ldots, K)$. La formule du lemme 4 devient alors: 


$$
\frac{\Phi(L)}{\Pi_{i=1}^{p} \rho_{\imath}}-\Phi(K)=\sum_{i=1}^{p} \frac{\Phi\left(L-\rho_{i} K\right)}{\rho_{i} \Pi_{, j \neq i}\left(\rho_{j}-\rho_{i}\right)} .
$$

Mais $\Phi\left(L-\rho_{i} K\right)=\sum_{k=0}^{p}\left(-\rho_{i}\right)^{k} \tilde{\Phi}\left(L^{(p-k)}, K^{k}\right)$, (où l'on a posé $\tilde{\Phi}\left(L^{(p-k)}, K^{k}\right)=$ $\tilde{\Phi}(L, \ldots, L, K, \ldots, K)$, l'argument $L$ étant répété $p-k$ fois et $K$ l'étant $k$ fois). Le second membre de la formule du lemme 4 est donc égal à:

$$
\sum_{k=0}^{p}\left((-1)^{k} \tilde{\Phi}\left(L^{(p-k)}, K^{k}\right) \sum_{i=1}^{p} \frac{\rho_{i}^{k-1}}{\Pi_{j, j \neq i}\left(\rho_{j}-\rho_{\imath}\right)}\right),
$$

et la formule du lemme 3 implique celle du lemme 4.

Fin de la deuxième démonstration du théorème 1: On applique le lemme 4 , avec $\rho_{i}=\frac{\beta_{i}}{\alpha_{i}}$

\section{BIBLIOGRAPHIE}

[B1] R. Bott, Vector fields and characteristic numbers, Michigan Math. J., 14 (1967), $231-244$.

[B2] — A residue formula for holomorphic vector fields, J. Differential Geometry, 1 (1967), 311-330.

[B3] - Lectures on characteristic classes and foliations, Springer Lecture Notes, 279 (1972).

[BB] P. Baum et R. Bott, Singularities of holomorphic foliations, J. Differential Geometry, 7 (1972), 279-342.

[L1] D. Lehmann, Résidus des sous variétés invariantes d'un feuilletage singulier, Ann. Inst. Fourier, 41 fasc. 1 (1991), 211-258.

[L2] - Residues of higher order and holomorphic vector fields, Ann. Global Anal. Geom., 12 (1994), 109-122.

[S] M. Soares, Algebraic differential systems on $\mathbf{P}^{\mathrm{n}}(\mathbf{C})$, en cours de rédaction.

Francisco Gomez:

Dep. Algebra, Geometría, Topología Fac. Ciencias, Université de Málaga

Campus Teatinos, Ap. 59, 29080 Málaga, Espagne

Email:GOMEZ-RUIZ@CCUMA.UMA.ES Fax(34) 52132000

Daniel Lehmann:

GETODIM, CNRS, UA 1407 Université de Montpellier II

Case 051, Place E. Bataillon, 34095 Montpellier cedex, France

Email:LEHMANN@FRMOP22.bitnet Fax (33)67543079 